\title{
Z-plasty for Correction of Standing Cutaneous Deformity
}

\author{
Ross Tanis, MD; Julie A. Croley, MD; Josh Hammel, MD; Richard F. Wagner Jr, MD
}

Cutaneous head and neck reconstruction following Mohs micrographic surgery frequently presents the surgical dilemma of dog-ear formation during wound closure. Z-plasty corrects a dog-ear deformity without skin excision by recruiting tissue from the axis of the standing cone and redistributing it along another. We describe dog-ear correction using the Z-plasty technique.

Cutis. 2020;106:80-81

\section{Practice Gap}

Cutaneous head and neck reconstruction following Mohs micrographic surgery frequently presents the surgical dilemma of dog-ear formation during wound closure, often necessitating excision of additional tissue to correct the standing cone, which could pose the risk for an undesirable tension vector as well as encroachment upon additional cosmetic units or sensitive anatomic structures such as a free margin. A classic Z-plasty is a transposition flap (by definition, translocation of tissue laterally about a pivot point) that corrects a dogear deformity without skin excision by recruiting tissue from the axis of the standing cone and redistributing it along another.

\section{The Technique}

A classic Z-plasty is designed with 3 equal limb lengths $\left(<1 \mathrm{~cm}\right.$ each) at $60^{\circ}$ angles, abutting the pedicle of the rotation or advancement flap. The limbs can extend away from the pedicle of the flap to minimize vascular compromise.
In our patient, the theoretical standing cone was located at the lateral aspect of an $\mathrm{O}$ to $\mathrm{L}$ advancement flap (Figure 1). The 2 identical triangular flaps were elevated (Figure 2A), transposed around the pivot point (Figure 2B), and inset (Figure 3). The standing cone was corrected by redistribution of tissue without excision of additional

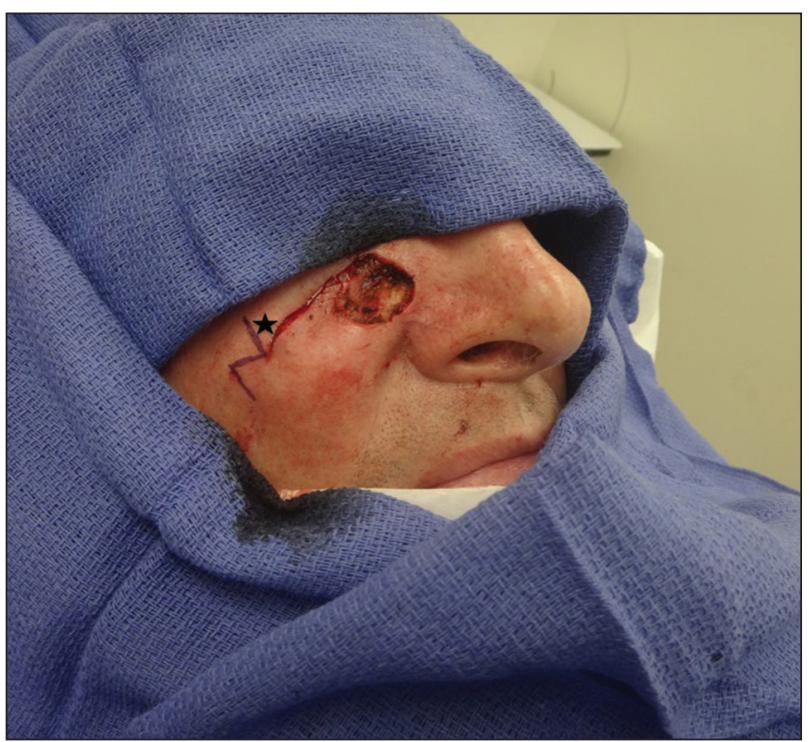

FIGURE 1. A Z-plasty abuts the lateral edge of an $O$ to $L$ advancement flap at the location of the future standing cone (star). It is designed as 3 limbs at $60^{\circ}$ angles, extending away from the pedicle of the flap.

From the Department of Dermatology, University of Texas Medical Branch, Galveston. 

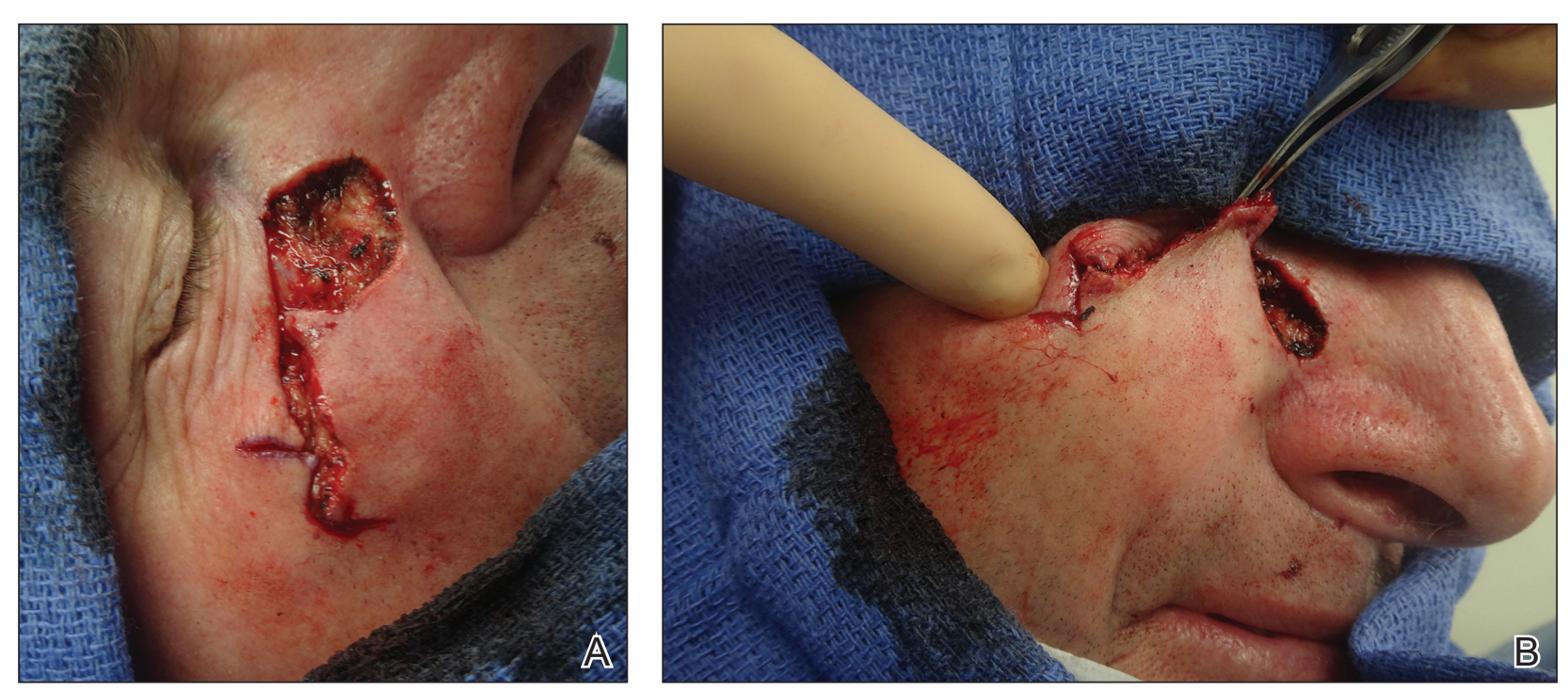

FIGURE 2. A and B, The flaps of the Z-plasty are elevated and transposed around the pivot point.

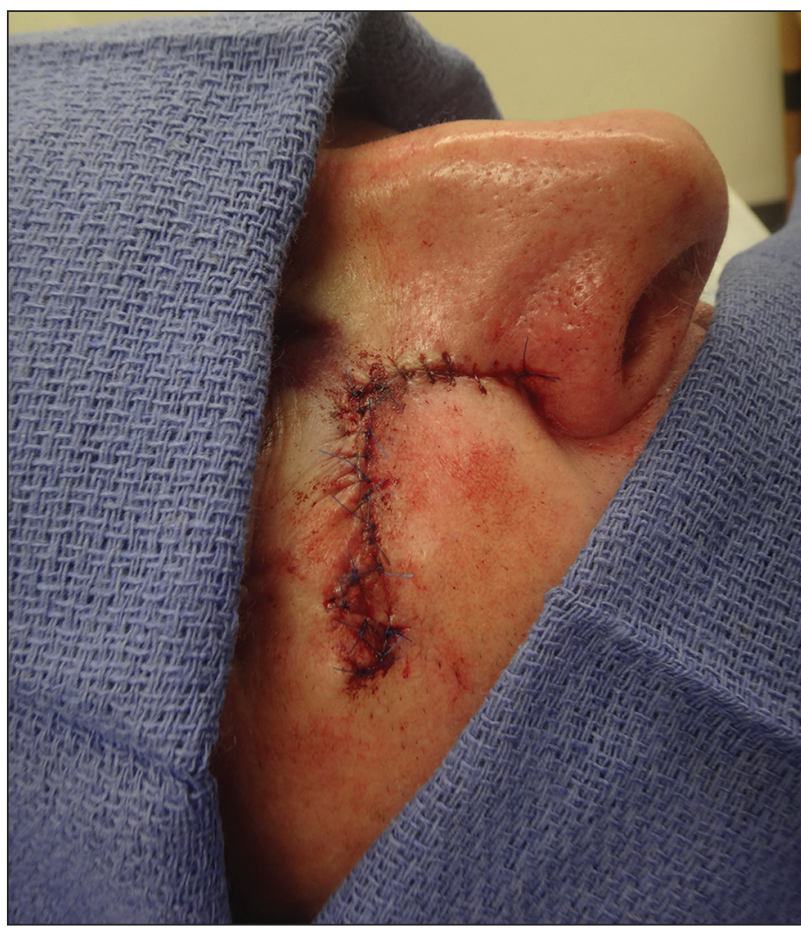

FIGURE 3. Flaps are inset without dog-ear formation.

tissue, resulting in a softer and thinner scar 2 weeks (Figure 4A) and 4 months (Figure 4B) postoperatively.

\section{Practice Implications}

This technique can be used to correct cones following primary wound repairs or flaps. The primary advantage of this technique for dog-ear correction is tissue sparing. Disadvantages include more complex surgical planning and longer scar length compared
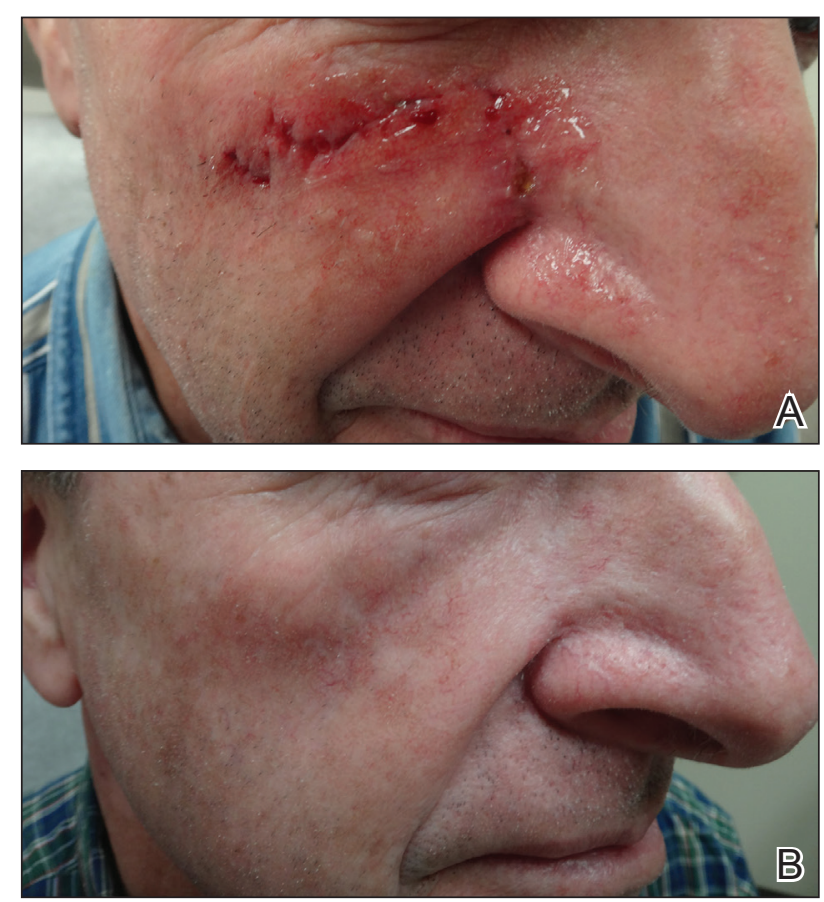

FIGURE 4. A, A soft thin scar was observed 2 weeks postoperatively. $\mathrm{B}$, Excellent cosmesis was achieved 4 months postoperatively.

to excisional corrective techniques. Additionally, Z-plasty requires more time to execute compared to simpler techniques. ${ }^{1,2}$

\section{REFERENCES}

1. Frodel JL, Pawar SS, Wang TD. Z-Plasty. In: Baker SR, ed. Local Flaps in Facial Reconstruction. 3rd ed. Elsevier; 2014:317-338.

2. Hundeshagen G, Zapata-Sirvent R, Goverman J, et al. Tissue rearrangements: the power of the Z-plasty. Clin Plast Surg. 2017; 44:805-812. 